\title{
3D printing in the preoperative planning and endovascular treatment of splenic artery aneurysm. Own clinical experience and literature review
}

\author{
Daniel G. Soliński ${ }^{1}$, Marcin Celer ${ }^{1}$, Krzysztof Dyś $^{1}$, Maciej Wiewióra ${ }^{2}$ \\ ${ }^{1}$ Regional Specialist Hospital, Research and Development Center, Wroclaw, Poland \\ ${ }^{2}$ Department of Cardiac, Vascular and Endovascular Surgery and Transplantology, Faculty of Medical Sciences in Zabrze, \\ Medical University of Silesia in Katowice, Poland
}

Videosurgery Miniinv 2022; 17 (1): 110-115

DOI: https://doi.org/10.5114/wiitm.2021.107765

\begin{abstract}
Splenic artery aneurysms (SAAs) are the most common visceral aneurysms. Endovascular treatment of SAAs is increasingly used. Appropriate preoperative imaging of aneurysms is crucial to treatment planning. The case of a patient with accidentally detected SAA on angio-CT examination was the basis for implementation of 3D printing to prepare an artery model. The 3D model made it easier to qualify for endovascular treatment of the SAA and helped to visualize its morphology. An excellent treatment effect was achieved. 3D printing provides an opportunity for better visualization of SAA anatomy, which has a direct impact on the choice of minimally invasive treatment method.
\end{abstract}

Key words: embolization, endovascular treatment, 3D printing, aneurysms, splenic artery, stent grafting.

\section{Introduction}

Splenic artery aneurysms (SAAs) were described for the first time in the literature in 1911 [1]. The normal diameter of the splenic artery varies individually and ranges from $0.43 \mathrm{~cm}$ to $0.49 \mathrm{~cm}$. An aneurysm is determined upon dilatation by $50 \%$ of the initial diameter [2]. SAAs are the most common visceral aneurysms and account for $60-70 \%$ of cases. SAAs are the third most common abdominal aneurysms after abdominal aortic aneurysms and iliac artery aneurysms. SAAs affect $0.8-1 \%$ of the population and are more common in women - approximately $78 \%$ [2, 3]. In the vast majority of cases, their development is asymptomatic. If left untreated, they can lead to serious complications associated with their rupture. The mortality of ruptured aneurysms of splenic arteries varies between $25 \%$ and $40 \%$ and increases to $75 \%$ in pregnant women [2]. Splenic artery aneurysms are most often accidentally detected during imaging examinations (ultrasound, computed tomography $(C T)$, magnetic resonance $(M R)$ ). In SAA follow-up, angio-CT and MR imaging are most commonly used. SAA treatment currently involves classic surgical procedures, which are considered the gold standard; laparoscopic procedures, which are less traumatic; and endovascular treatment, which in recent years has become increasingly popular due to low invasiveness and effectiveness and the low number of complications. Various techniques are used in endovascular treatment. The most commonly used techniques are embolization with embolizing spirals or liquid embolic materials and stent grafts [2-9]. The choice of treatment method and technique is difficult and is based on the type of aneurysm as well as its location and morphology. Diagnostic images 
are also of great importance in method selection, which is why innovative and easily accessible SAA imaging methods that will help select the best treatment technique need to be identified. 3D printing seems to meet the above criteria and complement the classic imaging methods by facilitating spatial reconstruction of SAAs along with the splenic artery [10]. Here, we present a patient with an SAA who qualified for endovascular treatment on the basis of an angio-CT examination and a 3D-printed model of the SAA based on this study and literature review.

\section{Aim}

The aim of this study was to indicate the possibility of using 3D printing technology in the minimally invasive, endovascular treatment of splenic artery aneurysms.

\section{Material and methods}

A 42-year-old patient suffered an injury to the left side of the body as a result of a fall from a low height. Due to persistent chest pain on the left side and suspected rib fracture, chest X-ray was performed. Unclear $X$-ray examination images and pain persisting for more than a few weeks required extending the diagnostics to $\mathrm{CT}$ examination. The $\mathrm{CT}$ scan accidentally detected a splenic artery aneurysm $24 \times 20 \times 19 \mathrm{~mm}$ in size. The examination was performed with 64-row, 128-layer, Optima CT660 ASIR, GE Healthcare, computed tomography. The size of the aneurysm qualified the patient for surgical treatment. To better visualize the neck of the aneurysm and evaluate the vessel, a preoperative model of the splenic artery was made using FFF 3D printing. The $3 \mathrm{D}$ print file was prepared from the angio-CT arterial phase DICOM data in open source license software. The arterial phase was marked based on the algorithm of threshold level segmentation and was manually corrected in each layer, trying to reproduce the course of the vessel as accurately as possible in accordance with the data obtained in the angio-CT study. After obtaining the virtual 3D model, a uniform wall thickness of $0.5 \mathrm{~mm}$ was determined, and the contour in each layer was again re-adjusted to obtain a model with light that best imitated the patient's vessel (Photo 1). The data were exported to a file with an STL extension (Photo 2). Then, the print parameters were set in the Ultimaker Cura program. The model was made with the Ultimaker
$2+$ printer. A thermoplastic polyurethane elastomer of Shore 98A hardness was used. The height of the model layer was set at $0.15 \mathrm{~mm}$, trying to maintain a balance between good print quality and time. The estimated printing time calculated by Cura was $3 \mathrm{~h}$. Approximately $10 \mathrm{~g}$ of filament was used. Tangent supports were generated only between the model and the printer table. Internal supports were not generated because the geometry of the vessel and proper planning of model placement on the printer table enabled the desired shape of the 3D model to be obtained. The material was selected for its mechanical properties, i.e., strength, flexibility across the entire hardness range, good back-strain properties, resistance to cracking and tearing. The model was printed on a $1: 1$ scale. The support material was removed manually.

\section{Results}

Six months after the injury, the patient was admitted to the hospital for endovascular treatment. After the patient was prepared for the procedure, right common femoral artery (RCFA) access was used to perform selective angiography of the celiac trunk and splenic artery. A $6 \times 50 \mathrm{~mm}$ Gore Viabahn stent graft was implanted in the splenic artery at the height of the aneurysm. Due to discrete leakage into the aneurysm sac, the aneurysm was also embolized with embolization spirals. Eleven Ev3 Concerto spirals were used for embolization. Control angiography showed normal flow through the splenic artery, lack of flow in the aneurysm sac and no loss of contrast enhancement in the spleen. RCFA puncture was performed with the Abbott StarClose SE VascularClosure percutaneous closure system. A few hours after the procedure, the patient did not report any complaints, and the next day she was discharged home in a good general condition, with the recommendation of anticoagulation and anti-aggregation therapy. Further controls were recommended at the vascular surgery outpatient clinic.

\section{Discussion}

3D printing is used in various fields of medicine [11-13]. In order to analyze the subject, a literature review was conducted using the PubMed, Epistemonikos, EBSCO and Google Scholar databases. There are reports on the use of 3D printing of hollow, preoperative models of intracranial vessel 

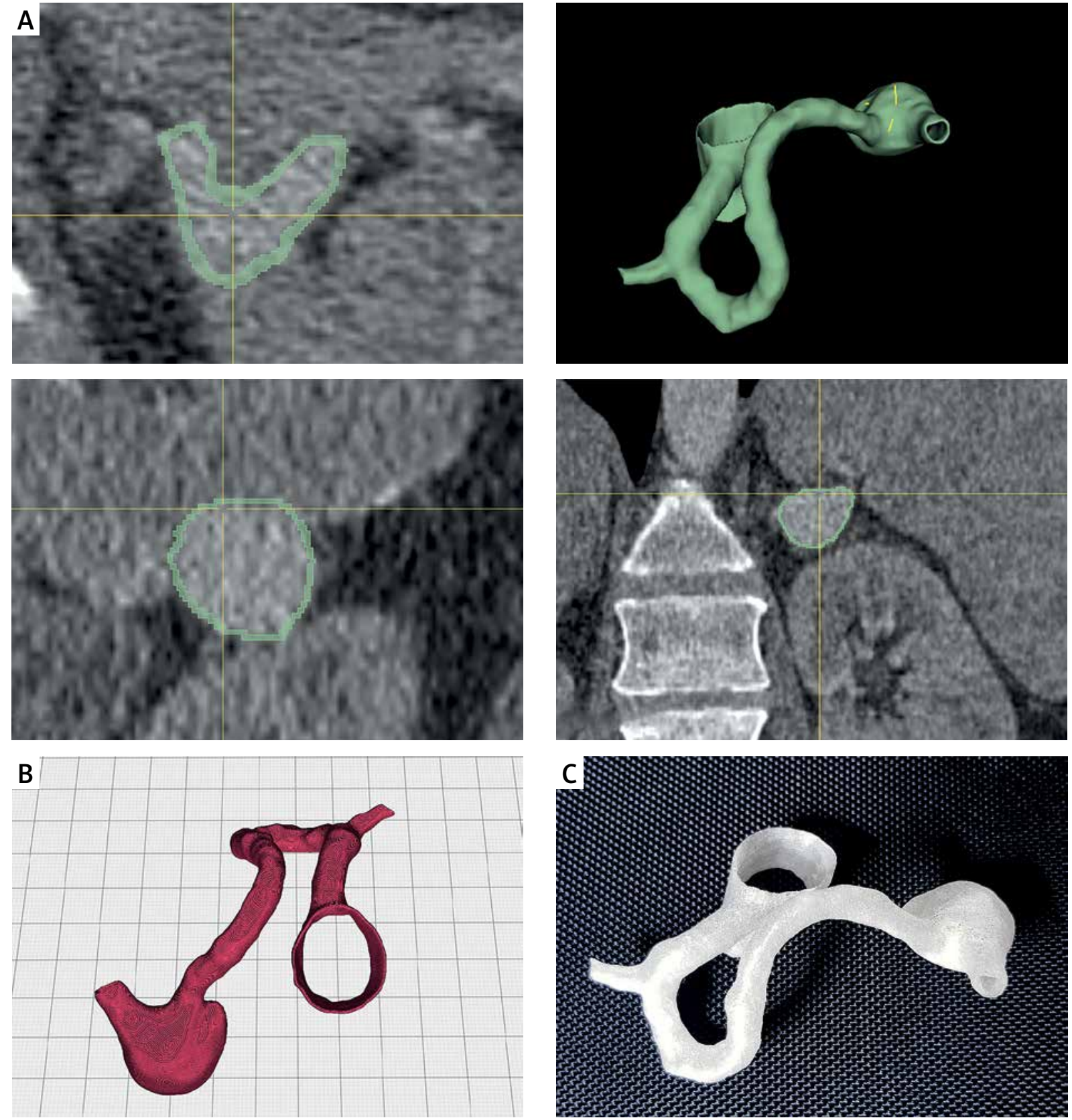

Photo 1 . Based on the angio-CT examination (A), a preoperative 3D model of the splenic artery aneurysm was prepared on a $1: 1$ scale $(\mathbf{B}, \mathbf{C})$

aneurysms to prepare for endovascular treatment. $3 \mathrm{D}$ printing was used in them as a technology supporting the production of silicone models. In addition, 3D printing is increasingly used in clinical applications of cardiovascular diseases, especially congenital heart disease, the treatment of aortic aneurysms and dissections, for simulation and training of needle puncture, the use of an externalized transapical guidewire in thoracic aneurysms repair, and to optimize computed tomography examination protocols. A significant part of the available literature is based on single case reports [14-17]. In the treatment of renal artery aneurysms (RAA), in 2 cases, 3D printing was used to plan classic surgical aneurysm management $[18,19]$ In the third case, the authors, on the basis of 3D printing, performed 

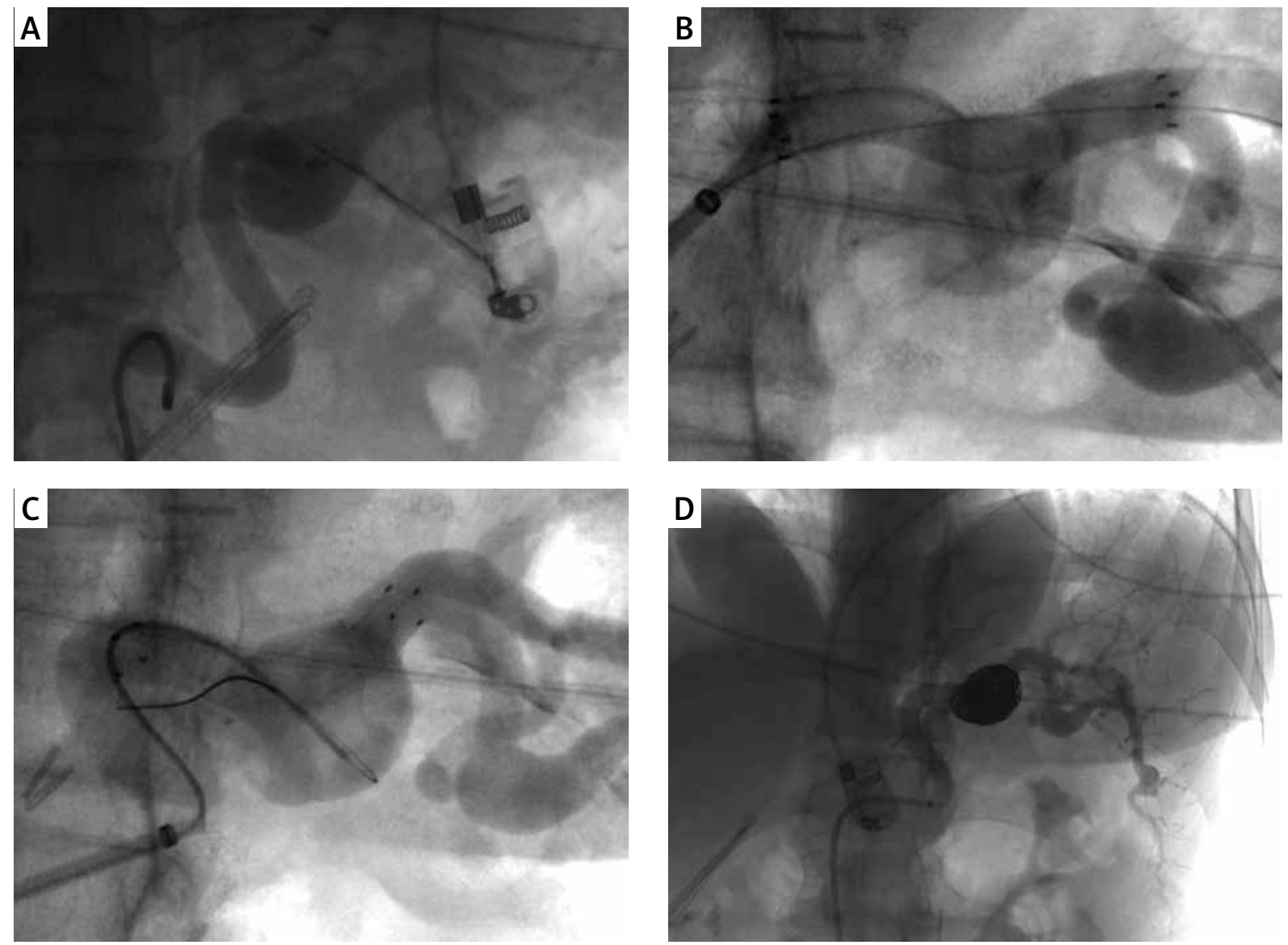

Photo 2. After visualization of the splenic artery and aneurysm on angiography (A), a Gore Viabahn stent graft was implanted (B). During the procedure, discrete leakage of the aneurysm sac was revealed. Additionally, the aneurysm was embolized with Ev3 Concerto embolization spirals (C), obtaining normal flow through the splenic artery, no lack of contrast with the spleen, and thus the planned effect of the procedure (D)

endovascular treatment of RAA, significantly reducing the invasiveness of the procedure [20]. So far, only two publications refer to the possibility of using 3D printing in the treatment of SAA. The first paper discusses only the technical aspects of printing and its quality [10]. In the second publication, printing was used in clinical practice, demonstrating its utility [21]. However, the implementation of 3D printing for the preoperative planning of endovascular procedures in visceral aneurysms is not common due to complex vessel geometry and technical limitations during model development [21]. Holographic technologies can significantly speed up the assessment of the patient's vascular anatomy by visualizing a virtual 3D model [22]. However, 3D printing has an advantage in the possibility of conducting bench tests, in which it is necessary to make real models, which may influence the decision on the selection of the size and type of the stent graft, including its fenestration [23, 24]. The supporting material that can help achieve complex shapes can be difficult to remove when printing with a single extruder printer. Generating an insoluble support material inside the model limits the possibility of fitting surgical tools by creating barriers that do not occur anatomically in the patient. Insufficient transparency of the model may also limit its use in preoperative equipment selection. On the other hand, previous studies have confirmed that 3D models of splenic artery aneurysms are highly precise and very well reproduce the results of imaging examinations [10]. In addition, $3 \mathrm{D}$ printing using the FFF method represents quite cheap and available technology. Open source software does not generate additional license costs, and the quality of printouts from FFF desktop printers is sufficient to obtain accurate spatial 3D prototypes. 
A wide range of thermoplastic materials allows the printing of rigid, flexible or completely transparent models. A general problem in the literature is the lack of a detailed analysis of the materials used for printing. Materials such as polylactic acid, nylon and acrylonitrile-butadiene styrene are most often used in FFF printing technology [10]. The simulation of the procedure on 3D models provides the possibility of manual training and transfer of pushability, torquability and trackability of endovascular tools and are assessed as needed in endovascular training [25]. Making models of hard, inelastic materials that do not reflect the properties of soft tissues prevents accurate pre-operative selection of endovascular instruments. From our point of view, the condition for bench tests on visceral vessels, including SA, is to obtain a model that reflects the mechanical properties of vessels. During the procedure, the SA is modeled by a rigid guidewire and expanded stent graft, and then after the guidewire is removed, it changes its geometry, at least partially returning to the original shape, which can lead to unexpected leakages into the SAA bag and even to repositioning of the stent graft. The bench test can help predict possible leakage only in the case of strain of the model wall under the guidewires' stiffness, catheters and endovascular equipment to a level comparable to the possible deformation of the actual vessel wall. Preferably a model should be placed in a closed artificial vascular system with the circulatory and pulsating fluid. After analyzing the model, we found that the use of high-hardness polymers, including TPU98A, is not a good basis for the anatomical SAA models, especially in terms of benchmark tests. In the case of self-preparation of 3D printing, the time from obtaining the CT scan to the finished 3D model, thus reproducing the morphology of the aneurysm, may be less than $6 \mathrm{~h}$. The advancement of FFF 3D printing technology and the introduction of dual extruder printers offer the prospect of even faster prototyping. Additionally, junction thermoplastic materials with water-soluble support materials should significantly reduce the problems associated with the complex geometry of visceral vessels. Endovascular treatment is becoming the method of choice in the treatment of aneurysms, including those of visceral arteries, due to the effectiveness of this method, the less invasive procedure for patients, the lack of need to open the abdominal cavity, and the shortening of the length of hospital stay [5]. It also allows most patients to maintain anatomical vessel continuity, in contrast to classical methods, in which reconstruction of the splenic artery after aneurysm excision is not always possible. Thorough preoperative knowledge of the splenic artery anatomy, especially of the aneurysm, is very important for successful endovascular treatment. The specific anatomy of the splenic artery and its often sinuous course can be a technical problem for performing endovascular surgery, as well as aneurysm morphology, length and neck bends. Computed tomography does not always allow for a clear assessment of the changes in single-plane cross-sections. Automatically generated virtual 3D models are at risk of algorithm errors and do not allow for preoperative accurate anatomy analysis with the use of surgical instruments. All these limitations can be compensated by expanding diagnostics using a volumetric and corporeal printed model.

\section{Conclusions}

The use of 3D printing to create 3D models of visceral artery aneurysms makes it easier to visualize their morphology and anatomy, which directly translates into selection of the best treatment method. The extension of an operator's preparation for a procedure with 3D-printed vascular system analysis could shorten the time of the procedure, reduce equipment consumption and accelerate the training of new doctors, which would not only result in economic benefits but also reduce potential negative complications that may occur due to long exposure to radiation or high contrast and medicinal doses. Due to the predominant benefits of 3D-printed vessel models, more frequent use of 3D printing in the preoperative diagnostics of complicated visceral artery aneurysms should be considered.

\section{Conflict of interest}

The authors declare no conflict of interest.

\section{References}

1. Taylor RS, Teacher JH. Two cases of aneurysm of the splenic artery. Glasgow Med J 1911; 75: 249-56.

2. Kassem MM, Gonzalez L. Splenic Artery Aneurysm. StatPearls. Treasure Island (FL) StatPearls Publishing 2020.

3. Ouchi T, Kato N, Nakajima K, et al. Splenic artery aneurysm treated with endovascular stent grafting: a case report and review of literature. Vasc Endovascular Surg 2018; 52: 663-8. 
4. Mkangala AM, Liang H, Dong XJ, et al. Safety and efficacy of conservative, endovascular bare stent and endovascular coil assisting bare stent treatments for patients diagnosed with spontaneous isolated superior mesenteric artery dissection. Videosurgery Miniinv 2020; 15: 608-19.

5. Hosn MA, Xu J, Sharafuddin M, Corson JD. Visceral artery aneurysms: decision making and treatment options in the new era of minimally invasive and endovascular surgery. Int J Angiol 2019; 28: 11-6.

6. Li ES, Mu JX, Ji SM, et al. Total splenic artery embolization for splenic artery aneurysms in patients with normal spleen. World J Gastroenterol 2014; 20: 555-60.

7. Martinelli O, Giglio A, Irace L, et al. Single-center experience in the treatment of visceral artery aneurysms. Ann Vasc Surg 2019; 60: 447-54.

8. Wojtaszek M, Lamparski K, Wnuk E, et al. Selective occlusion of splenic artery aneurysms with the coil packing technique: the impact of packing density on aneurysm reperfusion correlated between contrast-enhanced MR angiography and digital subtraction angiography. Radiol Med 2019; 124: 450-9.

9. Zhu C, Zhao J, Yuan D, et al. Endovascular and surgical management of intact splenic artery aneurysm. Ann Vasc Surg 2019; 57: 75-82.

10. Takao H, Amemiya S, Shibata E, Ohtomo K. 3D printing of preoperative simulation models of a splenic artery aneurysm: precision and accuracy. Acad Radiol 2017; 24: 650-3.

11. Mitsouras D, Liacouras P, Imanzadeh A, et al. Medical 3D printing for the radiologist. Radiographics 2015; 35: 1965-88.

12. Marro A, Bandukwala T, Mak W. Three-dimensional printing and medical imaging: a review of the methods and applications. Curr Probl Diagn Radiol 2016; 45: 2-9.

13. Rengier F, Mehndiratta A, von Tengg-Kobligk $\mathrm{H}$, et al. 3D printing based on imaging data: review of medical applications. Int J Comput Assist Radiol Surg 2010; 5: 335-41.

14. Sun Z. Clinical applications of patient-specific 3D printed models in cardiovascular disease: current status and future directions. Biomolecules 2020; 10: 1577.

15. Rynio P, Falkowski A, Witowski J, et al. Simulation and training of needle puncture procedure with a patient-specific 3D printed gluteal artery model. J Clin Med 2020; 9: 686.

16. Rynio P, Kazimierczak A, Jedrzejczak T, Gutowski P. A 3D printed aortic arch template to facilitate decision-making regarding the use of an externalized transapical wire during thoracic endovascular aneurysm repair. Ann Vasc Surg 2019; 54: 336. e5-e8.

17. Jędrzejczak T, Rynio P, Lewandowski M, Kazimierczak A. Externalized transapical guidewire technique after artificial aortic valve replacement during complete endovascular aortic arch repair. Videosurgery Miniinv 2021; 16: 227-33.

18. Holzem KM, Jayarajan S, Zayed MA. Surgical planning with three-dimensional printing of a complex renal artery aneurysm. J Vasc Surg Cases Innov Tech 2018; 4: 19.

19. Lin JC, Myers E. Three-dimensional printing for preoperative planning of renal artery aneurysm surgery. I Vasc Surg 2016; 64: 810.
20. Soliński D, Celer M, Dyś K, Witkiewicz W. The use of 3D printing in the treatment of renal artery aneurysms. ECR 2020. doi: 10.26044/ecr2020/C-09049.

21. Itagaki MW. Using 3D printed models for planning and guidance during endovascular intervention: a technical advance. Diagn Interv Radiol 2015; 21: 338-41.

22. Rynio P, Witowski J, Kamiński J, et al. Holographically-guided endovascular aneurysm repair. J Endovasc Ther 2019; 26: 544-7.

23. Kazimierczak A, Rynio P, Jędrzejczak T, et al. Utility of dual longitudinal diameter-reducing ties in aortic arch thoracic endovascular aortic repair. Adv Interv Cardiol 2019; 15: 485-8.

24. Rynio P, Kazimierczak A, Jedrzejczak T, Gutowski P. A 3-Dimensional printed aortic arch template to facilitate the creation of physician-modified stent-grafts. J Endovasc Ther 2018; 25: 554-8.

25. Mafeld S, Nesbitt C, McCaslin J, et al. Three-dimensional (3D) printed endovascular simulation models: a feasibility study. Ann Transl Med 2017; 5: 42.

Received: 2.02.2021, accepted: 29.03.2021. 